\title{
OPEN A 52 weeks dupilumab treatment for moderate to severe atopic dermatitis in Korea: long-term efficacy and safety in real world
}

\author{
Dong Hyek Jang ${ }^{1}$, Seok Jae Heo ${ }^{2}$, Hyung Don Kook ${ }^{1}$, Dong Heon Lee ${ }^{1}$, Hye Jung Jung ${ }^{1}$, \\ MiYeon Park ${ }^{1}$ \& Jiyoung Ahn (i] $1{ }^{1}$
}

Previously, we have reported short term effectiveness and safety of dupilumab in Korea. In this study, we are trying to report the long-term effectiveness and safety of dupilumab in Korea. Ninety-nine patients with moderate to severe $A D$ were analyzed. They were evaluated using Eczema Area and Severity Index (EASI), Numerical Rating Scale (NRS), Patient Oriented Eczema Measure (POEM), and Dermatology Quality of Life Index (DLQI) at baseline, week 16, 32 and 52 . Efficacy outcomes showed higher improvement at 52 weeks compared with 16 weeks; high percentual reductions in EASI (88.1\%), peak pruritus NRS (65.6\%), POEM (67.2\%), and DLQI (69.0\%) compared to baseline. Proportion of patients achieving EASI 75 and 90 were $90.2 \%$ and 53.7\%. POEM and DLOI had high correlation with clinical measured outcomes. In the analysis for the factors affecting achievement of EASI 90, female gender (OR 2.5), eosinophilia (OR 0.2) and elevated LDH (OR 0.07) were significantly associated. Most frequent adverse events included facial erythema (19.2\%) and conjunctivitis (17.2\%), which were mild/moderate and resolved during treatment. In conclusion, dupilumab treatment for 52 weeks in Korean patients with moderate-to-severe AD confirmed long term effectiveness and safety.

Atopic dermatitis $(\mathrm{AD})$ is a representative chronic inflammatory systemic disease ${ }^{1}$, and various treatments have been developed depending on the stage ${ }^{2,3}$. From classical systemic immunosuppressants to newly developed biologics and small molecules that inhibit various cytokines that contribute to the immune response, there are many ongoing studies ${ }^{4-6}$. Among them, dupilumab, a monoclonal antibody that blocks the shared receptor component for interleukin (IL)-4 and IL-13, is a first biological agent approved for the treatment of patients with moderate to severe AD. Until recently, it has been most actively used and considered as a game changer in the treatment of severe AD.

Dupilumab has shown efficacy and safety in existing phase 3 and 4 clinical trials ${ }^{8}$ and long-term effectiveness in various real-world studies ${ }^{9-14}$. Previous data from Korea have shown significant improvements and safety in moderate-to-severe AD patients treated with dupilumab for 16 weeks ${ }^{15}$. However, data for effectiveness and safety for long-term treatment with dupilumab in $\mathrm{AD}$ patients in real world is limited. Therefore, we aimed to evaluate the long-term effectiveness and safety of 52 weeks of treatment with dupilumab in moderate to severe $\mathrm{AD}$ patients in Korea and investigate the prognostic factors affecting the treatment outcome.

\section{Methods}

Study design. This retrospective analysis was conducted through electronic medical records of patients with moderate to severe AD treated with dupilumab at National Medical Center in Korean from September 2018 to December 2020. Patients were assigned to dupilumab $600 \mathrm{mg}$ on day 1, then to dupilumab $300 \mathrm{mg}$ every 2 weeks, with the exception of 9 patients who received dupilumab 300 mg every 3 or 4 weeks. Along with dupilumab, all patients were treated with topical calcineurin inhibitor (TCI). Some patients who have been previously under treatment with systemic immunosuppressants gradually reduced dose in order to prevent abrupt discontinuation and received initially concomitant treatment with dupilumab and the previously used systemic immunosuppressants, including systemic steroids. After sufficient tapering, all concomitant treatments except dupilumab were discontinued within 2 weeks. This study was approved by the Institutional Review Board of the National Medical Center, and was conducted in accordance with the principles of the Declaration of Helsinki. 
Patients and efficacy outcomes. Eligible patients had an Eczema Area and Severity Index (EASI) score of 16 or higher and were over 18 years old who were diagnosed with $\mathrm{AD}$ based on the diagnostic criteria ${ }^{16}$ by a dermatologist. Patients were assessed for efficacy outcomes and measured for laboratory tests at baseline, 16, 32, and 52 weeks after treatment as follows: Efficacy outcomes; EASI, peak pruritus Numerical Rating Scale (NRS) , Patient Oriented Eczema Measure (POEM), and Dermatology Quality of Life Index (DLQI ${ }^{17-20}$, Laboratory tests; Whole Blood Count, Liver Function Test, Renal Pannel, Lipid Profile, Immunoglobulin E (IgE), Total Eosinophil Count (TEC), and Lactate Dehydrogenase (LDH).

Statistical analysis. Statistical analysis was performed using R 3.6.3 version (R Foundation for Statistical Computing, Vienna, Austria). The changes in efficacy outcomes and laboratory tests between the baseline and 16,32 , and 52 weeks of treatment were analyzed using the linear mixed model with discrete time points. Correlation analysis between efficacy outcomes at 52 weeks and laboratory test results was performed to identify the factors most relevant to the patient's quality of life. The differences in efficacy outcomes after 52 weeks according to the level of IgE, TEC, and $\mathrm{LDH}$ at each measurement point were investigated. In addition, multivariative logistic regression analysis was performed using age, sex, disease onset, initial combined treatment, IgE, TEC, and $\mathrm{LDH}$ as independent variables to determine whether there are factors affecting the achievement of EASI 90 after 52 weeks of treatment. Statistical significance was determined at a p value less than 0.05 .

Informed consent. Need of informed consent is waived by the Institutional Review Board of National Medical Center.

\section{Results}

Demographics. Of 99 patients, 58 men and 41 women had an average age of 30.92 years; 48 (48.48\%) were in their $20 \mathrm{~s}$ to $30 \mathrm{~s}$, while 38 (38.38\%) were in their $30 \mathrm{~s}$ and $40 \mathrm{~s}$. One third of patients (36\%) had a family history of allergic diseases and more than half $(57.58 \%)$ had personal history of other allergic diseases. Among them, allergic rhinitis, allergic conjunctivitis and asthma were the most frequent $(85.96 \%, 29.82 \%$, and $21.05 \%$, respectively). About half (55.56\%) of patients had known allergies mostly caused by house dust mite (96.36\%). Topical steroid was the most frequent previous treatment used $(92.93 \%)$ followed by TCI $(68.69 \%)$. Treatment with systemic steroid and non-steroid immunosuppressive drugs accounted for $86.89 \%$ and $61.61 \%$ of the subjects, respectively. Cyclosporine was the most common non-steroid immunosuppressant (86.89\%). The proportion of oriental medicine and folk remedies were $52.53 \%$ and $27.27 \%$, respectively, with oriental medicine in more than half of the cases (Table 1).

Most patients $(82.83 \%)$ were treated with dupilumab with TCI only over the 52 weeks. For patients initiating dupilumab in combination with other systemic drug for tapering, the initial combination treatment was cyclosporine in $7 \%$, methotrexate in $6 \%$, and oral steroid in $4 \%$.

Efficacy of treatment. Baseline value of efficacy outcomes. Outcome measures used in this study have been validated for atopic dermatitis and are recommended by $\mathrm{HOME}^{21}$. The mean baseline values for EASI, pruritus NRS, POEM, and DLQI are presented in Table 2, and correspond to severe disease.

Changes in efficacy outcomes at 16, 32 and 52 weeks. All outcome measures reduced from the most severe levels towards mild values overtime. EASI values reduced by $75 \%$ at week 16 (mean 7.6 ) and $88 \%$ at week 52 (mean 3.5). Pruritus NRS reduction was $59 \%$ at week 16 (mean 3.4) and $66 \%$ at week 52 (mean 2.8). POEM score (0-28) improved by $57 \%$ at week 16 (mean 10) and $67 \%$ at week 52 (mean 7.3 ) and DLQI improved by $60 \%$ at week 16 (mean 8.9) and 69\% at week 52 (mean 6.5, representing small impact in life). All differences were statistically significant vs baseline values $(\mathrm{p}<0.001)$ (Table 2$)$.

EASI 75 and EASI 90 at 52 weeks. EASI 75 was achieved by $56.10 \%, 86.59 \%$, and $90.24 \%$ of patients at 16, 32, and 52 weeks, respectively, and EASI 90 was achieved in $9.76 \%, 35.37 \%$, and $53.66 \%$ of patients at 16,32 , and 52 weeks, respectively (Fig. 1a,b).

Changes in laboratory tests at 16, 32 and 52 weeks. Compared to the baseline values, there was a significant decrease in IgE, TEC, and LDH at 16, 32, and 52 weeks. There was a 53.64\% decrease for IgE from 3964.05 to $1837.87,44.82 \%$ for TEC from 931.67 to 514.13 , and $30.15 \%$ for LDH from 280.81 to 196.14 (Table 3). No significant changes in other laboratory findings were observed.

Correlations between efficacy outcomes. All variables showed significant correlations, except that between EASI and DLQI. The correlation coefficients of EASI with pruritus NRS, POEM and DLQI were 0.32, 0.24 , and 0.18 , respectively. On the other hand, about NRS, POEM, and DLQI, which are subjective outcomes compared to EASI, the correlation coefficients between pruritus NRS and POEM, pruritus NRS and DLQI, and POEM and DLQI were $0.66,0.62$, and 0.78 , respectively with the correlation coefficient between POEM and DLQI being the highest (Fig. 2).

Relationship between laboratory markers and treatment response. The improvement in EASI after 52 weeks was significantly lower in the group with high LDH than in the group with low LDH at baseline, 16 and 32 weeks, respectively. Although there were significant differences in each outcome for LDH at 52 weeks, 


\begin{tabular}{|c|c|c|}
\hline Type & Variables & Mean (min, max) or count (\%) \\
\hline \multirow{28}{*}{ Demographics } & Age, years & $30.92(18.00-53.00)$ \\
\hline & $10-20$ & $4(4.04 \%)$ \\
\hline & $20-30$ & $48(48.48 \%)$ \\
\hline & $30-40$ & $38(38.38 \%)$ \\
\hline & $40-50$ & $7(7.07 \%)$ \\
\hline & $50-60$ & $2(2.02 \%)$ \\
\hline & Gender & \\
\hline & Male & $58(58.59 \%)$ \\
\hline & Female & $41(41.41 \%)$ \\
\hline & Occupation & \\
\hline & Yes & $68(68.69 \%)$ \\
\hline & Marriage & \\
\hline & Yes & $19(19.19 \%)$ \\
\hline & Disease onset & \\
\hline & Adult exacerbation & $6(6.06 \%)$ \\
\hline & Childhood & $93(93.94 \%)$ \\
\hline & Family history of allergy & \\
\hline & Yes & $36(36.36 \%)$ \\
\hline & Allergy history & $57(57.58 \%)$ \\
\hline & Allergic conjunctivitis & $17(29.82 \%)$ \\
\hline & Allergic rhinitis & $49(85.96 \%)$ \\
\hline & Asthma & $12(21.05 \%)$ \\
\hline & Known allergy & $55(55.56 \%)$ \\
\hline & House dust mite & $53(96.36 \%)$ \\
\hline & Mold & $19(34.55 \%)$ \\
\hline & Food & $5(9.09 \%)$ \\
\hline & Cat & $6(10.91 \%)$ \\
\hline & Dog & $3(5.45 \%)$ \\
\hline \multirow{14}{*}{ History of treatment } & Topical treatment & \\
\hline & Topical steroid & $92(92.93 \%)$ \\
\hline & Topical calcineurin inhibitor & $68(68.69 \%)$ \\
\hline & Systemic treatment & \\
\hline & Systemic steroid & $65(65.66 \%)$ \\
\hline & Immunosuppressant & $61(61.61 \%)$ \\
\hline & Cyclosporine & $53(86.89 \%)$ \\
\hline & Methotrexate & $6(9.84 \%)$ \\
\hline & Mycophenolate mofetil & $1(1.64 \%)$ \\
\hline & Azathioprine & $1(1.64 \%)$ \\
\hline & Phototherapy & $20(20.20 \%)$ \\
\hline & Immunotherapy & $13(13.13 \%)$ \\
\hline & Oriental medicine & $52(52.53 \%)$ \\
\hline & Folk remedy & $27(27.27 \%)$ \\
\hline
\end{tabular}

Table 1. Baseline demographics and history of treatment $(n=99)$.

it was difficult to consider the results as statistically appropriate because there was a large difference in sample size between the comparison groups (Table 4). There were no significant differences for IgE and TEC.

Variables associated with achieving EASI 90. Under adjustment to the baseline EASI, we performed logistic regression analysis for each variable. In achieving EASI 90, female sex showed an odds ratio (OR) of $2.509(\mathrm{p}=0.036)$ compared to males. About TEC, eosinophilia at the baseline and 16 weeks was not statistically significant, while eosinophilia at 32 and 52 weeks showed OR as $0.250(\mathrm{p}=0.005)$ and $0.218(\mathrm{p}=0.005)$, respectively. $\mathrm{LDH}$ showed statistical significance at all time points, especially at 52 weeks with an OR of 0.068 $(\mathrm{p}=0.037)$ (Table 5).

In addition, we separately performed multivariative analysis to identify the effects of several variables at once under adjustment to the baseline EASI. It was also confirmed that female sex (OR 3.321, p=0.033) and high LDH level (OR 0.252, $\mathrm{p}=0.036$ ) were factors associated with achieving EASI 90. (Table 6). 


\begin{tabular}{|l|l|c|c|c|}
\hline & Baseline & After 16 weeks & After 32 weeks & After 52 weeks \\
\hline Mean EASI score \pm SD & $30.02 \pm 10.84$ & $7.61 \pm 4.77^{*}$ & $4.66 \pm 3.25^{\star}$ & $3.50 \pm 2.88^{*}$ \\
\hline Mean percent change \pm SD in EASI & & $74.90 \pm 11.57$ & $84.49 \pm 9.05$ & $88.14 \pm 8.56$ \\
\hline Mean NRS score \pm SD & $8.37 \pm 1.72$ & $3.36 \pm 2.13^{*}$ & $3.24 \pm 2.07^{\star}$ & $2.80 \pm 1.82^{*}$ \\
\hline Mean percent change \pm SD in NRS & & $59.37 \pm 5.42$ & $59.51 \pm 28.93$ & $65.55 \pm 23.05$ \\
\hline Mean POEM \pm SD & $23.73 \pm 4.35$ & $9.99 \pm 6.33^{*}$ & $9.00 \pm 6.19^{*}$ & $7.34 \pm 5.86^{*}$ \\
\hline Mean percent change \pm SD in POEM & & $56.56 \pm 27.34$ & $59.95 \pm 29.34$ & $67.21 \pm 29.34$ \\
\hline Mean DLQI \pm SD & $22.37 \pm 5.27$ & $8.93 \pm 6.59^{*}$ & $7.60 \pm 6.86^{*}$ & $6.54 \pm 5.74^{*}$ \\
\hline Mean percent change \pm SD in DLQI & & $59.59 \pm 29.23$ & $61.01 \pm 29.51$ & $69.02 \pm 27.61$ \\
\hline
\end{tabular}

Table 2. Change in efficacy outcomes after 16, 32 and 52 weeks-all showed a significant decrease. SD, standard deviation; EASI, Eczema Area and Severity Index; NRS, Numerical Rating Scale; POEM, patientoriented eczema measure; DLQI, Dermatology Life Quality Index. ${ }^{\star} \mathrm{p}$ value $<0.05$, $\mathrm{P}$ value calculated by linear mixed model with discrete time points.

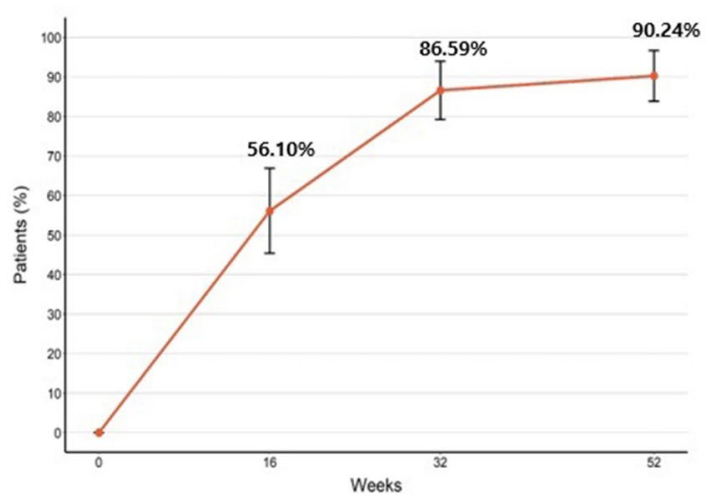

b

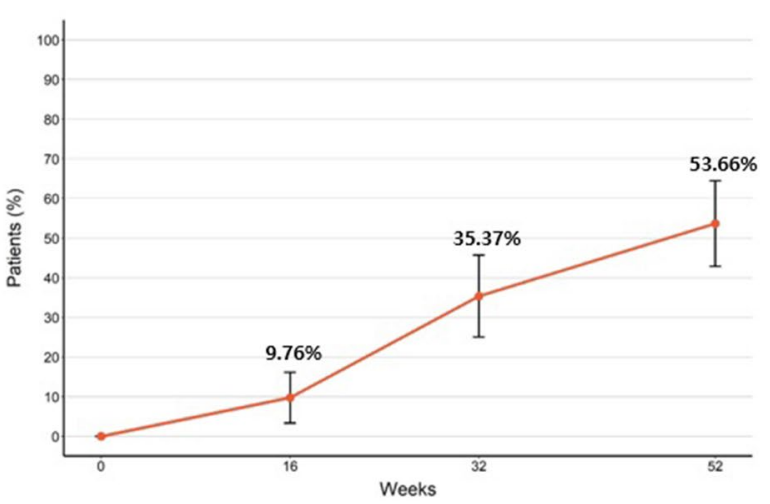

Figure 1. The proportion of (a) EASI 75 and (b) EASI 90 at baseline, after 16, 32 and 52 weeks.

\begin{tabular}{|l|l|c|l|l|}
\hline & Baseline & After 16 weeks & After 32 weeks & After 52 weeks \\
\hline Mean serum total Ig E \pm SD & $3964.05 \pm 6411.23$ & $2601.08 \pm 3843.12^{\star}$ & $1960.34 \pm 3020.99^{\star}$ & $1837.87 \pm 3043.89^{\star}$ \\
\hline Mean serum TEC \pm SD & $931.67 \pm 805.49$ & $737.54 \pm 846.80^{\star}$ & $618.93 \pm 628.58^{\star}$ & $514.13 \pm 487.55^{\star}$ \\
\hline Mean serum LDH \pm SD & $280.81 \pm 85.32$ & $219.19 \pm 65.02^{\star}$ & $212.12 \pm 63.21^{\star}$ & $196.14 \pm 43.80^{\star}$ \\
\hline
\end{tabular}

Table 3. Change in laboratory test after 16, 32 and 52 weeks-all showed a significant decrease. Ig E, immunoglubulin E; TEC, total eosinophil count; $\mathrm{LDH}$; lactate dehydrogenase. ${ }^{\star} \mathrm{p}<0.05$, p value calculated by linear mixed model with discrete time points.

Adverse events associated with treatment. The main adverse effects associated with dupilumab treatment were facial erythema and conjunctivitis. A total of 19 patients $(19.19 \%)$ developed facial erythema associated with dupilumab. After dupilumab treatment, 17 patients developed facial erythema, while pre-existing facial erythema became exacerbated in two patients. Most of the facial erythema improved after maintenance with TCI treatment, while one patient who showed no response to TCI treatment improved with oral itraconazole $200 \mathrm{mg}$ once daily for 5 weeks. Six patients developed persistent facial erythema unresponsive to treatment, but considered not serious enough to discontinue dupilumab treatment. One patient was diagnosed with systemic lupus erythematosus (SLE); the erythema improved after increasing the dosing interval of dupilumab and cooperating with the rheumatologist.

Conjunctivitis developed in 17 patients (17.17\%) after administration of dupilumab. Most cases improved with increasing the dosing interval or ophthalmic consultation, five patients showed persistent conjunctivitis. None of the patients discontinued from treatment due to conjunctivitis because they thought that the improvement of $\mathrm{AD}$ was important and conjunctivitis gradually improved. 

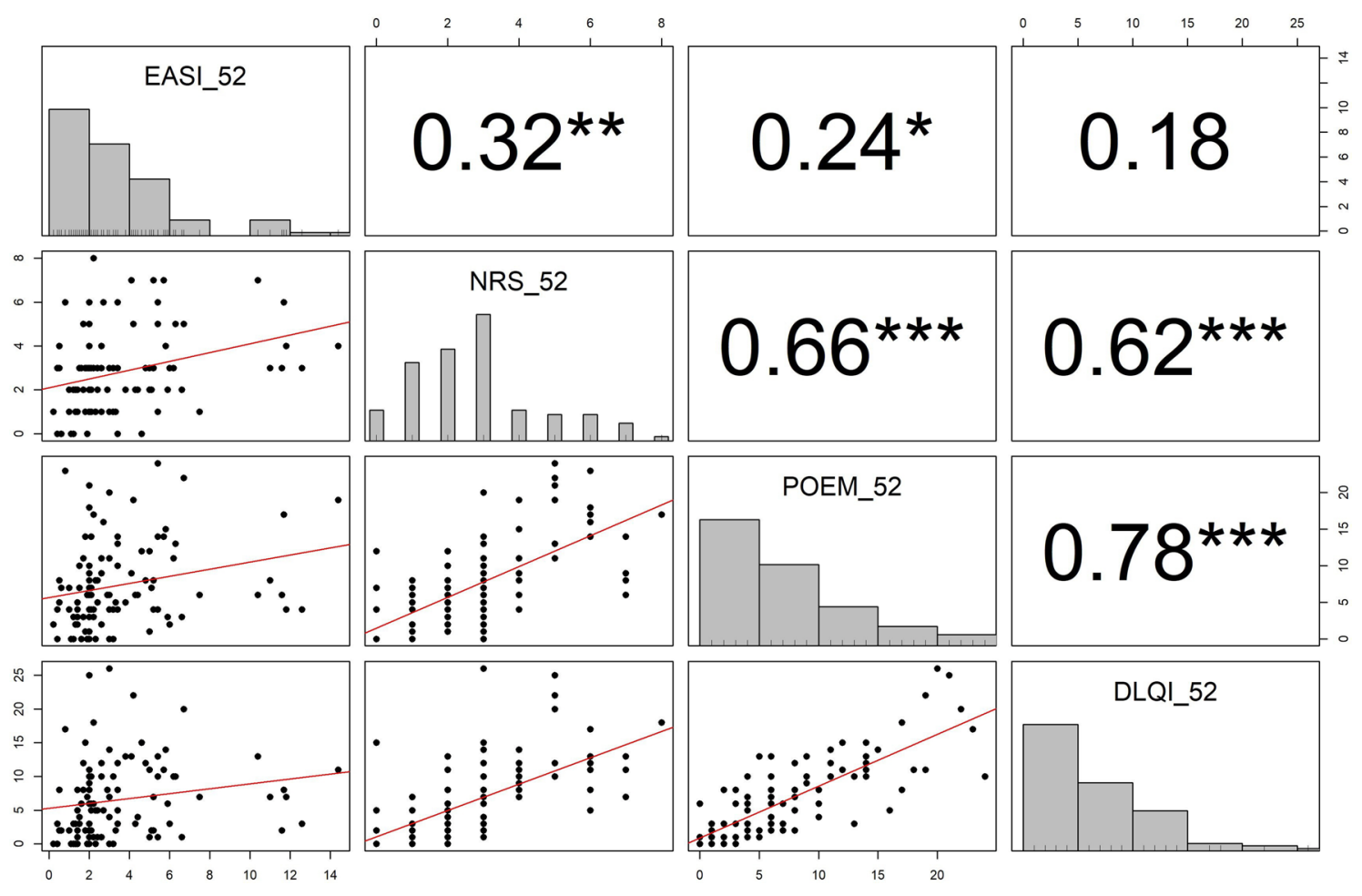

Figure 2. The correlation analysis between EASI, NRS, POEM, and DLQI at 52 weeks. All p-values less than $0.05,0.01$, or 0.001 are summarized as ${ }^{\star}, * *$, or ${ }^{\star * *}$, respectively.

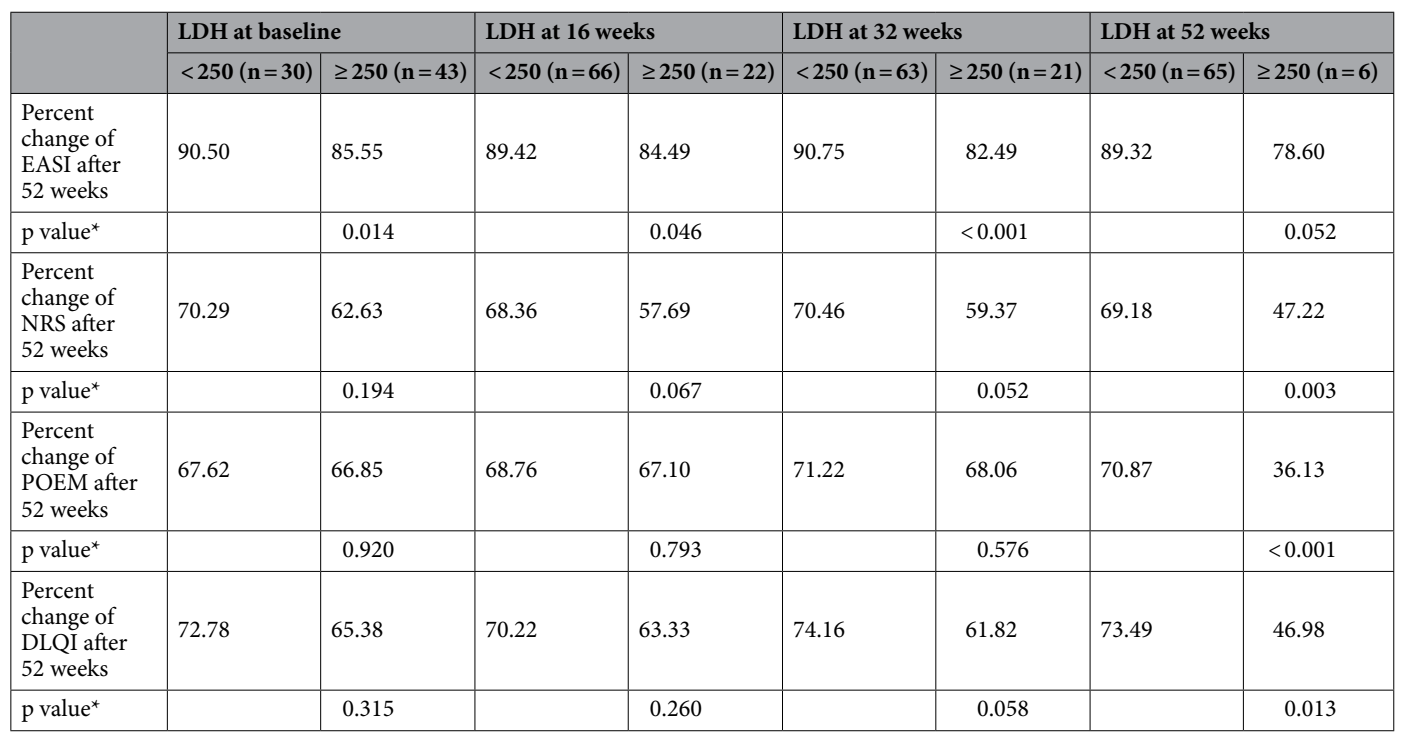

Table 4. The difference of efficacy, according to LDH-elevated LDH at baseline, 16 and 32 weeks showed inadequate treatment response in EASI. ${ }^{*} \mathrm{p}$ values were obtained by two sample independent t-test.

\section{Discussion}

While real-world studies on the long-term effectiveness and safety of dupilumab treatment have been conducted and published in various countries ${ }^{9-14}$, there were limited data on its long-term results in Korean AD patients despite our previous study of dupilumab treatment for 16 weeks. In this study, we assessed long-term effectiveness and safety of 52 weeks of continuous treatment with dupilumab in Korean patients with moderate-to-severe AD. In addition, we analyzed the correlation between efficacy outcomes and factors affecting the therapeutic effect ${ }^{15}$. Dupilumab significantly improved clinical factors and symptoms of AD in moderate-to-severe patients over the 52-week treatment period, confirming its long-term therapeutic effect and safety. 


\begin{tabular}{|l|l|l|l|l|l|l|l|}
\hline Variables & OR & $\mathbf{9 5 \%} \mathbf{C I}$ & P value & Variables & OR & $\mathbf{9 5 \%}$ CI & P value \\
\hline Age over 30 & 0.627 & $0.275-1.405$ & 0.259 & TEC $>500$ at baseline & 0.621 & $0.234--1.606$ & 0.330 \\
\hline Sex (female) & 2.509 & $1.077-6.089$ & 0.036 & TEC $>500$ at 16 weeks & 0.477 & $0.197-1.115$ & 0.093 \\
\hline Onset (adult) & 1.004 & $0.174-5.801$ & 0.996 & TEC $>500$ at 32 weeks & 0.250 & $0.090-0.641$ & 0.005 \\
\hline Concomitant treatment & 0.384 & $0.114-1.158$ & 0.100 & TEC $>500$ at 52 weeks & 0.218 & $0.071-0.605$ & 0.005 \\
\hline $\begin{array}{l}\text { Concomitant treatment with cyclo- } \\
\text { sporine }\end{array}$ & 0.852 & $0.378-1.907$ & 0.697 & LDH $>250$ at baseline & 0.259 & $0.085-0.719$ & 0.012 \\
\hline IgE $>100$ at baseline & 5.188 & $0.733-104.917$ & 0.151 & LDH $>250$ at 16 weeks & 0.264 & $0.079-0.788$ & 0.022 \\
\hline IgE $>100$ at 16 weeks & 1.186 & $0.304-4.748$ & 0.804 & LDH $>250$ at 32 weeks & 0.136 & $0.033-0.446$ & 0.002 \\
\hline IgE $>100$ at 32 weeks & 0.610 & $0.175-1.991$ & 0.420 & LDH $>250$ at 52 weeks & 0.068 & $0.003-0.600$ & 0.037 \\
\hline IgE $>100$ at 52 weeks & 0.311 & $0.087-1.023$ & 0.062 & & & & \\
\hline
\end{tabular}

Table 5. The multivariate logistic regression analysis about each variable associated with achieving EASI 90 - female, TEC > 500 at 32 and 52 weeks, and $\mathrm{LDH} \geq 250$ at all time points showed significant difference, respectively. Each analysis was performed about the effect of each variable under adjustment to the baseline EASI, respectively.

\begin{tabular}{|l|l|l|l|}
\hline Variables & OR & 95\% CI & P value \\
\hline Age (over 30) & 0.708 & $0.242-2.036$ & 0.522 \\
\hline Sex (female) & 3.321 & $1.141-10.648$ & 0.033 \\
\hline Disease onset (adult onset) & 1.456 & $0.105-18.670$ & 0.765 \\
\hline Concomitant treatment & 0.451 & $0.094-1.871$ & 0.287 \\
\hline Concomitant treatment with cyclosporine & 0.802 & $0.279-2.303$ & 0.680 \\
\hline TEC $\geq 500$ at baseline & 1.077 & $0.303-4.062$ & 0.910 \\
\hline LDH $\geq 250$ at baseline & 0.252 & $0.064-0.879$ & 0.036 \\
\hline
\end{tabular}

Table 6. The multivariate logistic regression analysis about variables associated with achieving EASI 90female and $\mathrm{LDH} \geq 250$ at baseline showed significant difference. This analysis was performed with the variables presented in the table at once under adjustment to the baseline EASI.

Among the baseline values, DLQI was found to be exceptionally high in Korea compared to other studies including that of Japan ${ }^{8-14}$. As mentioned in previous studies, no clear improvements were seen in Korean AD patients although various treatments were used to improve their lesions. This is supported by the relatively high proportion of subjects who used oriental medicine and folk remedy as their past treatments ${ }^{22}$.

Overall, this study showed a higher efficacy than the existing clinical trials and other real-world studies ${ }^{8-14}$; $88.58 \%$ of patients achieved significant improvements in EASI at week 52 in this study, compared to $78.3 \%$ in LIBERTY AD CHRONOS, and 76.38\% in United Kingdom (UK) real-world data. Although outcomes regarding NRS, POEM, and DLQI were similar to those of UK, the results of this study were effective considering that the data from the UK were relatively from mild patients ${ }^{8,9}$. Compared with the results of a study conducted in Japan, our study showed greater efficacy. Particularly, there was a remarkable difference in that the study in Japan resulted in the reduction by $76.5 \%$ in EASI after 12 months ${ }^{14}$. In addition, the achievement of EASI 75 and 90 was $90.24 \%$ and $53.66 \%$ in this study, compared to $65 \%$ and $51 \%$ in LIBERTY AD CHRONOS or $63 \%$ and $29 \%$ in the UK, respectively. The following reasons were considered for the higher percentual of improvement in our data to that of existing clinical trials and real-world studies in other countries. Because of the easy accessibility to hospitals in Korea, the majority of patients regularly visited the hospital every two weeks, and the dermatologist could check their condition every time. Whenever their condition worsened, further treatment was immediately initiated. For example, in the case of a patient with a worsening of eosinophilia, which was thought as a poor prognosis, short-term systemic steroid treatment was applied to support the therapeutic effect of dupilumab. In addition to dupilumab treatment, all patients basically used TCI. Patients were fully informed about the importance of using TCI before initiating treatment and continued to use TCI despite the improvements shown with dupilumab. This combination of TCI and dupilumab is thought to increase the therapeutic effect by acting as a "proactive treatment" $23-25$ even after AD has improved. In January 2020, National Health Insurance Service provided coverage for dupilumab treatment in Korea. For insurance coverage, it should satisfy strict conditions with regular dosing intervals and prove the therapeutic effect of dupilumab. If AD worsened, it could no longer be covered by health insurance. Therefore, patients themselves made efforts to use moisturizers or avoid exposure to known allergens; we considered this behavior as "adherence" to the treatment. Based on these results, we could identify the importance of "adherence" and "proactive treatment".

In the correlation analysis, there was a significantly high correlation between NRS, POEM, and DLQI, which are indicators of subjective symptoms, but there was a low correlation with EASI which is considered as a relatively objective marker. This is consistent with previous studies ${ }^{15,26}$ that suggested the importance of subjective 

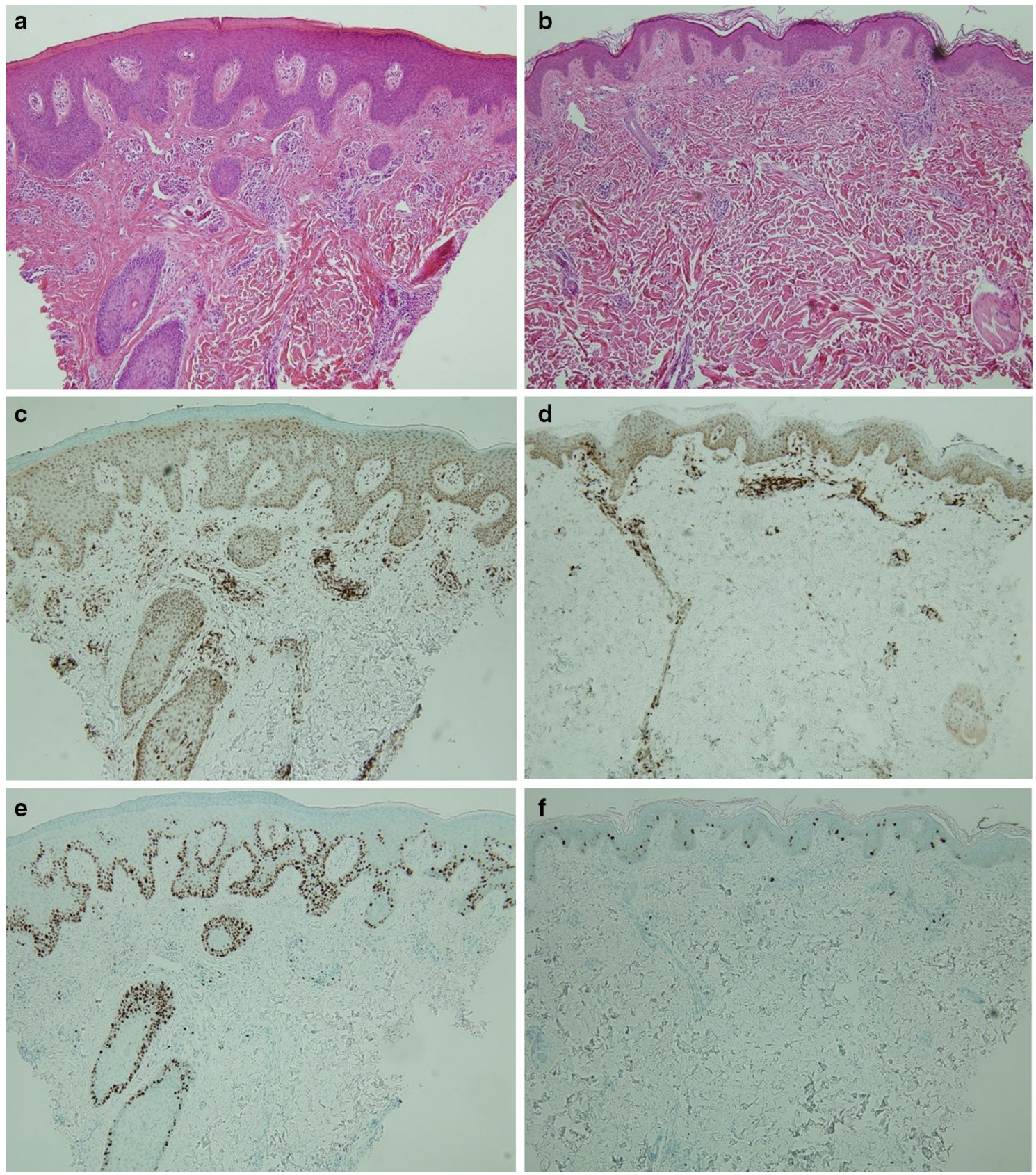

Figure 3. Skin biopsys at baseline and after 16 weeks to be compared by (a, b) Hematoxylin and eosin, (c, d) Cluster of differentiation 3 (CD3), and (e, f) Ki-67. After 16 weeks, the epidermal thickness, irregular acanthosis, the expression of $\mathrm{T}$ cells and ki-67 expression were markedly decreased.

symptoms, in addition to objective symptoms, and particularly shows the usefulness of POEM in evaluating the patient's quality of life.

As suggested in previous studies, our long-term study confirmed that women had a good treatment response, and eosinophilia and high LDH level were associated with a poor treatment response. The real-world study results in which females showed better therapeutic effects than males were also consistent with this study ${ }^{27}$. Compliance and drug distribution according to body weight were considered as the reasons why women have better prognosis. Previous studies have suggested that women have higher treatment compliance than men in AD, contact dermatitis, and psoriasis ${ }^{28-30}$. Therefore, these data may have influenced the 'adherence' that appeared during treatment. Although weight and BMI cannot be measured in all patients, if women were assumed to have a lower body weight than men, then the distribution of drugs per unit body weight was higher in females, since the treatment regimen was performed with the same dosage and interval. A clinical trial of dupilumab in children with severe AD also showed a therapeutic effect according to body weight ${ }^{31}$. This is considered meaningful, although it should be verified through validated and controlled trials. 
It has been previously suggested that high eosinophilia may be associated with poor therapeutic efficacy of dupilumab $^{32}$. It has also been observed that in these patients, short-term systemic steroids can lower eosinophils and raise the effect of dupilumab. One study suggested that high LDH level is associated with decreased effect of dupilumab under adjustment to the baseline $\mathrm{EASI}^{33}$. An ongoing study attempted to investigate the degree of influence of markers known to be related to disease activity in AD on the therapeutic effects of dupilumab treatment. In addition to IgE, TEC and LDH, a total of 18 markers including soluble IL 2 receptor, IL-13, IL-22, IL-31, and thymic stromal lymphopoietin were included ${ }^{34}$. Recently, various therapeutic agents that block each stage of the pathophysiology of AD have been developed ${ }^{4-6}$ and are considered as a good treatment option. Therefore, predictive biomarkers are important as they are used to identify patients who are more responsive to certain therapeutic agents that show good therapeutic effects. In this study, biomarkers that affect the prognosis of dupilumab treatment were identified, which is expected to maximize the effect of dupilumab treatment.

Studies about skin biopsy performed before and after dupilumab treatment showed the significant decrease in cytokines associated with type 2 inflammation, such as IL-13 and IL-31, and T cells and dendritic cells, and in epidermal thickness ${ }^{35,36}$. In this study, skin biopsy was performed twice at the same site, before initiating of dupilumab treatment and at 16 weeks (Fig. 3). The epidermal thickness and irregular acanthosis markedly decreased after treatment, and the expression of $\mathrm{T}$ cells in the dermis and ki 67, a proliferation marker at the base of the epidermis, was also significantly reduced. Since biopsy was not performed on all patients, increased number of samples in future studies could provide meaningful results for comparison.

Facial erythema is a side effect that has not been specifically reported in existing clinical trials, even though it may have a possible connection with dupilumab treatment in the clinical setting. The cause is not simple; there are various possibilities from exacerbation of existing atopic lesions, rebound by tapering or discontinuation of existing therapeutic agents, allergic contact dermatitis, seborrheic dermatitis positive reaction to Malassezia yeast, to unknown side effects of dupilumab. In rare cases reported, acute cutaneous lupus erythematosus is likely to be stimulated by dupilumab in our SLE patient ${ }^{37-43}$. Therefore, it is necessary to investigate the epidemiologic and clinical characteristics of facial erythema that occurred after dupilumab treatment.

In the previous 16-weeks study ${ }^{15}$, there was a significantly lower rate of conjunctivitis in contrast to the results of existing clinical trials and other real-world studies conducted during the same period. However, the frequency of conjunctivitis increased to a similar degree as in other studies over 16-52 weeks of the treatment period. One study suggested that $\mathrm{AD}$ patients treated with dupilumab had an increased risk of developing conjunctivitis ${ }^{44}$. In particular, there was high risk of conjunctivitis in patients with severe AD prior to initiation of treatment, and high frequency in cases of prior conjunctivitis or increased thymus and activation-regulated chemokines, IgE, or eosinophils before initiation of treatment ${ }^{45-47}$. Although the etiology of conjunctivitis in dupilumab-treated patients remains unknown, the following causes are considered. The reduced ocular cytokine due to dupilumab forms an environment which favors the growth of Demodex mites ${ }^{48}$. Eosinophilia after dupilumab treatment ${ }^{46}$, increase in downstream activity of OX40 ligand ${ }^{49}$, inhibition of IL-13 and indirect decreased production of mucin in the goblet cells of the conjunctiva are also potential factors that affect conjunctivitis ${ }^{50}$. In addition, it has been suggested that there is no need to discontinue dupilumab or adjust the interval to control conjunctivitis. Accordingly, patients with prior conjunctivitis are not contraindicated for dupilumab, but ophthalmology treatment is recommended prior to initiation of dupilumab ${ }^{44}$. Conjunctivitis is an important side effect related to dupilumab treatment. Therefore, identification of symptoms by the dermatologist is crucial, and the treatment of conjunctivitis should be properly performed through cooperation with the ophthalmologist.

\section{Conclusion}

Retrospective real-world studies are very important to define the effectiveness of new drugs. In this long term 1 year study including 99 Korean patients, we confirmed the continuous therapeutic benefit of dupilumab, with most patients responding to treatment. We identified potential factors associated with response such as female sex, baseline eosinophilia, and high LDH levels were Additionally, this study confirmed that "adherence" and "proactive treatment" can increase the therapeutic effect of dupilumab in real life. This study is useful in the understanding of long-term dupilumab treatment in Asian patients with moderate to severe AD.

Received: 13 September 2021; Accepted: 24 November 2021

Published online: 07 December 2021

\section{References}

1. Silverberg, J. I. \& Kantor, R. The role of interleukin 4 and/or 13 in the pathophysiology and treatment of atopic dermatitis. Dermatol. Clin. 35, 327-334 (2017).

2. Wollenberg, A. et al. Consensus-based European guidelines for treatment of atopic eczema (atopic dermatitis) in adults and children: part I. J. Eur. Acad. Dermatol. Venereol. 32, 657-682 (2018).

3. Wollenberg, A. et al. Consensus-based European guidelines for treatment of atopic eczema (atopic dermatitis) in adults and children: part II. J. Eur. Acad. Dermatol. Venereol. 32, 850-878 (2018).

4. Ahn, J. et al. Emerging systemic therapeutic biologics and small molecules for atopic dermatitis: how to decide which treatment is right for your patients. J. Allergy. Clin. Immunol. Pract. 9, 1449-1460 (2021).

5. Ahn, J., Choi, Y. \& Simpson, E. L. Therapeutic new era for atopic dermatitis: part 1-biologics. Ann. Dermatol. 33, 1-10 (2021).

6. Ahn, J., Choi, Y. \& Simpson, E. L. Theratpeutic new era for atopic dermatitis: part 2-small molecules. Ann. Dermatol. 33, 101-107 (2021).

7. Simpson, E. L. et al. Two phase 3 trials of dupilumab versus placebo in atopic dermatitis. N. Eng. J. Med. 375, 2335-2348 (2016).

8. Blauvelt, A. et al. Long-term management of moderate-to-severe atopic dermatitis with dupilumab and concomitant topical corticosteroids (LIBERTY AD CHRONOS): a 1-year, randomized, double-blinded, placebo-controlled, phase 3 trial. Lancet 389, 2287-2303 (2017).

9. Sears, A. V. et al. Real-world effectiveness and tolerability of dupilumab in adult atopic dermatitis: a single-centre, prospective 1 -year observational cohort study of the first 100 patient treated. Br. J. Dermatol. 184, 755-757 (2021). 
10. Ruiz-Villaverde, R. et al. Fifty-two week follow-up safety and effectiveness results of dupilumab treatment of moderate-to-severe atopic dermatitis from a retrospective, multicentric series. Dermatol. Ther. 32, e12931. https://doi.org/10.1111/dth.12931 (2019).

11. Ariens, L. F. M. et al. Dupilumab shows long-term effectiveness in a large cohort of treatment-refractory atopic dermatitis patients in daily practice: 52-Week results from the Dutch BioDay registry. J. Am. Acad. Dermatol. 84, 1000-1009 (2021).

12. Silverberg, J. I. et al. Real-world persistence with dupilumab among adults with atopic dermatitis. Ann. Allergy. Asthma. Immunol. 126, 40-45 (2021).

13. Casado, B., Sanchez, R. \& Sierra, G. Real long-term data of dupilumab in atopic dermatitis. Rev. Ofil. Ilaphar. 30, 25-27 (2020).

14. Uchida, H. et al. One-year real-world clinical effectiveness, safety, and laboratory safety of dupilumab in Japanese adult patients with atopic dermatitis: a single-center retrospective study. J. Am. Acad. Dermatol. 84, 547-550 (2021).

15. Jang, D. H. et al. Retrospective study of dupilumab treatment for moderate to severe atopic dermatitis in Korea: efficacy and safety of dupilumab in real-world practice. J. Clin. Med. 9, 1982 (2020).

16. Hanifin, J. M. et al. Guidelines of care for atopic dermatitis, developed in accordance with the American Academy of Dermatology (AAD)/American Academy of Dermatology Association "Administrative Regulations for Evidence Based Clinical Practice Guidelines". J. Am. Acad. Dermatol. 50, 391-404 (2004).

17. Hanifin, J. M. et al. The Easi Evaluator Group. The eczema area and severity index (EASI). Exp. Dermatol. 10, 11-18 (2001).

18. Yosipovitch, G. et al. Validation of the peak pruritus numerical rating scale. Br. J. Dermatol. 181, 761-769 (2019).

19. Spuls, P. I. et al. Patient-Oriented Eczema Measure (POEM), a core instrument to measure symptoms in clinical trials: a Harmonising Outcome Measures for Eczema (HOME) statement. Br. J. Dermatol. 176, 979-984 (2017).

20. Finlay, A. Y. \& Khan, G. K. Dermatology Life Quality Index (DLQI) - a simple practical measure for routine clinical use. Clin. Exp. Dermatol. 19, 210-216 (1994).

21. Chalmers, J. R. et al. Report from the fourth international consensus meeting to harmonize core outcome measures for atopic eczema/dermatitis clinical trials (HOME initiative). Br. J. Dermatol. 175, 69-79 (2016).

22. Jung, H. J. et al. Survey of disease awareness, treatment behavior and treatment satisfaction in patients with atopic dermatitis in Korea: a multicenter study. J. Dermatol. 45, 1172-1180 (2018).

23. Thomas, B. Atopic dermatitis. Ann. Dermatol. 22, 125-137 (2010).

24. Wollenberg, A. \& Ehmann, L. M. Long term treatment concepts and proactive therapy for atopic eczema. Ann. Dermtol. 24, 253-260 (2012).

25. Fukuie, T. et al. Potential preventive effects of proactive therapy on sensitization in moderate to severe childhood atopic dermatitis: a randomized, investigator-blinded, controlled study. J. Dermatol. 43, 1283-1292 (2016)

26. Ahn, J. et al. Correlation of atopic dermatitis with measurement tools in Korean patients: a retrospective study Indian. J. Dermatol. Venereol. Leprol. 86, 738-740 (2020).

27. Olesen, C. M. et al. Treatment of atopic dermatitis with dupilumab: experience from a tertiary referral centre. J. Eur. Acad. Dermatol. Venereol. 33, 1562-1568 (2019).

28. Murota, H. et al. Characterization of socioeconomic status of Japanese patients with atopic dermatitis showing poor medical adherence and reasons for durg discontinuation. J. Dermatol. Sci. 79, 279-287 (2015).

29. Noiesen, E. et al. Gender differences in topical treatment of allergic contact dermatitis. Acta. Derm. Venereol. 89, 79-110 (2009).

30. Colombo, D. et al. Gender medicine and psoriasis. World. J. Dermatol. 3, 36-44 (2014).

31. Paller, A. S. et al. Efficacy and safety of dupilumab with concomitant topical corticosteroids in children 6 to 11 years old with severe atopic dermatitis: a randomized, double-blinded, placebo-controlled phase 3 trial. J. Am. Acad. Dermatol. 83, 1282-1293 (2020).

32. Ahn, J., Bae, J. Y. \& Seo, S. J. A retrospective case series hypereosinophilia during dupilumab treatment: Hypereosinophilia could be a pharmacogenetic biomarker of dupilumab. Dermatol. Ther. 33, e13847. https://doi.org/10.1111/dth.13847(2020) (2020).

33. Kato, A. et al. Higher baseline serum lactate dehydrogenase level is associated with poor effectiveness of dupilumab in the long term in patients with atopic dermatitis. J. Dermatol. 47, 1013-1019 (2020).

34. Nakahara, T. et al. Exploration of biomarkers to predict clinical improvement of atopic dermatitis in patients treated with dupilumab. Medicine 99, e22043. https://doi.org/10.1097/MD.0000000000022043 (2020).

35. Hamilton, J. D. et al. Dupilumab improves the molecular signature in skin of patients with moderate-to-severe atopic dermatitis. J. Allergy. Clin. Immunol. 134, 1293-1300 (2014).

36. Guttman-Yassky, E. et al. Dupilumab progressively improves systemic and cutaneous abnormalities in patients with atopic dermatitis. J. Allergy. Clin. Immunol. 143, 155-172 (2019).

37. De Wijs, L. E. M. et al. Clinical and histopathological characterization of paradoxical head and neck erythema in patients with atopic dermatitis treated with dupilumab: a case series. Br. J. Dermatol. 183, 745-749 (2020).

38. Jang, D. H. et al. Facial erythema after the treatment of dupilumab in SLE patient. Allergy. Asthma. Clin. Immunol. 16, 60 (2020).

39. De Beer, F. S. A. et al. Dupilumab facial redness: positive effect of itraconazole. J. Am. Acad. Dermatol. Case. Rep. 5, 888-891 (2019).

40. Waldman, R. A. et al. Characterizing dupilumab facial redness: a multi-institution retrospective medical record review. J. Am. Acad. Dermatol. 82, 230-232 (2020).

41. Yoseph, D. \& Sandra, M. Case report: first reported case of facial rash after Dupilumab therapy. Pract. Dermatol. 2018, 25-26 (2018).

42. Arnold, K. A., Treister, A. D. \& Lio, P. A. Dupilumab in the management of topical corticosteroid withdrawal in atopic dermatitis a retrospective case series. J. Am. Acad. Dermatol. Case. Rep. 4, 860-862 (2018).

43. Suresh, R. \& Murase, J. E. The role of expanded series patch testing in identifying causaility of residual facial dermatitis following initiation of dupilumab therapy. J. Am. Acad. Dermatol. Case. Rep. 4, 899-904 (2018).

44. Agnihortri, G., Shi, K. \& Lio, P. A. A clinician`s guide to the recognition and management of dupilumab-associated conjunctivitis. Drugs. R D 19, 311-318 (2019).

45. Akinlade, B. et al. Conjunctivitis in dupilumab clinical trials. Br. J. Dermatol. 181, 459-473 (2019).

46. Thyssen, J. P. et al. Incidence, prevalence, and risk of selected ocular disease in adults with atopic dermatitis. J. Am. Acad. Dermatol. 77, 280-286 (2017).

47. Uchio, E. et al. Systemic and local immunological features of atopic dermatitis patients with ocular complications. Br. J. Ophthalmol. 82, 82-87 (1998).

48. Thyssen, J. P. Could conjunctivitis in dupilumab treated atopic dermatitis patients be caused by colonization with Demodex and increased IL-17 levels?. Br. J. Dermatol. 178, 1220 (2018).

49. Simpson, E. L., Akinlade, B. \& Ardeleanu, M. Two phase 3 trials of dupilumab versus placebo in atopic dermatitis. N. Eng. J. Med. 376, 1090-1091 (2017)

50. Bakker, D. S. et al. Goblet cell scarcity and conjunctival inflammation during treatment with dupilumab in patients with atopic dermatitis. Br. J. Dermatol. 180, 1248-1249 (2019).

\section{Author contributions}

D.H.J.: writing-original draft (lead); investigation (equal), writing-review \& editing (equal). S.J.H.: formal analysis (lead); validation (lead); visualization (lead). H.D.K.: investigation (equal). D.H.L.: investigation (equal). H.J.J.: supervision (equal); investigation (equal). M.Y.P.: supervision (equal); investigation (equal). J.A.: conceptualization (lead); methodology (lead); supervision (lead); writing-review \& editing (lead). 


\section{Competing interests}

The authors declare no competing interests.

\section{Additional information}

Correspondence and requests for materials should be addressed to J.A.

Reprints and permissions information is available at www.nature.com/reprints.

Publisher's note Springer Nature remains neutral with regard to jurisdictional claims in published maps and institutional affiliations.

(1) Open Access This article is licensed under a Creative Commons Attribution 4.0 International License, which permits use, sharing, adaptation, distribution and reproduction in any medium or format, as long as you give appropriate credit to the original author(s) and the source, provide a link to the Creative Commons licence, and indicate if changes were made. The images or other third party material in this article are included in the article's Creative Commons licence, unless indicated otherwise in a credit line to the material. If material is not included in the article's Creative Commons licence and your intended use is not permitted by statutory regulation or exceeds the permitted use, you will need to obtain permission directly from the copyright holder. To view a copy of this licence, visit http://creativecommons.org/licenses/by/4.0/.

(C) The Author(s) 2021 